\title{
Advanced nurse practitioners; what do the team think?
}

\author{
Authors: Anuska Glendinning and David Walker
}

\section{Introduction}

The advanced nurse practitioner (ANP) is an established role within many hospitals and can be found in all aspects of health organisations. This new role has equipped nurses to take on more procedures as well as tasks traditionally associated with junior doctors, including the ability to diagnose and prescribe, while still retaining their foundation nursing roles. Previous research has focused on the ANP role within the community setting or emergency departments. This innovative study aimed to examine perceptions from the nursing and medical teams of an ANP working on a gastroenterology ward.

\section{Methods}

A qualitative study was conducted at the Royal United Hospital Bath using three separate focus groups, each comprising of five doctors (foundation year 1 to registrar), five nurses (grades 5-7) and six ANPs. Each focus group lasted between 40-60 minutes and consisted of a set format of questions to guide discussions and aid with moderation of the group (eg perceptions of the value of the ANP role within the ward environment, advantages of an ANP and how the ANP assists with a doctor's role). Transcripts of the interviews were analysed and the data was reduced into themes.

\section{Results and discussion}

Thematic analysis identified three positive themes related to the perception of an ANP working on a gastroenterology ward: (1) assisting with workload; (2) teamwork; and (3) leadership. It was highlighted that due to the ANP's understanding of the processes of patient flow, tasks were pre-empted and performed in advance, improving efficiency and reducing the workload of other team members. Within the analysis there was an overriding theme of consistency; quotes from doctors included, "the ANP is an absolute "life line" at the start of a new rotation. Their consistency allowed things not to get missed and acted as a safety net for us all, patients alike'. There were several sub-themes relevant to gastroenterology, particularly; 'sharing of applied skills and knowledge'. This was relevant for ascitic paracentesis, nasogastric (NG) tube insertion, implementation of the liver care bundle and application of gastroenterology algorithms for complex patients which included gastrointestinal (GI) bleeding.

\section{Conclusion}

The study demonstrated that an ANP is a great asset to a medical ward and a valued member of the team. They provide consistency within the department, to both the nursing and medical teams. This is deemed vital, due to the ongoing 4-monthly rotations of junior medical staff, which is perceived as disruptive to teamwork and affecting consistency of patient care. The results have also identified how the ANP role appears to be more than just a 'work gap' solving role, they are paramount in sharing skills and knowledge with junior doctors as well as contributing to and enhancing teamwork, which is essential in healthcare transformation.

\section{Conflict of interest statement}

None declared. 